\title{
Câncer na população feminina brasileira
}

\section{Cancer among women in Brazil}

\author{
Gulnar Azevedo e Silva Mendonça*
}

\begin{abstract}
MENDONÇA, G. A. e S. Câncer na população feminina brasileira. Rev. Saúde Pública, 27: 68-75, 1993. Objetivou-se mostrar a importância do câncer enquanto causa de morte e morbidade para a população feminina brasileira. Foram analisados os dados das estatísticas de mortalidade do Ministério da Saúde e os disponíveis nos registros de câncer de base populacional existentes nos municípios em Belém, Fortaleza, Recife, Goiânia, São Paulo e Porto Alegre (Brasil). Em 1986 o câncer foi responsável por $15,5 \%$ dos 6 bitos em mulheres acima de quinze anos no Brasil. Os cânceres de mama $\mathrm{e}$ útero representaram quase um terço desses óbitos. A comparação internacional mostrou que os coeficientes de incidência de câncer de colo de útero em Recife e Belém foram os mais altos do mundo e os de mama em Fortaleza e São Paulo são próximos dos encontrados nas regiōes de mais altas cifras como nos Estados Unidos e alguns países da Europa. São discutidos os principais fatores de risco para os cânceres mais prevalentes entre as mulheres brasileiras, guardando as diferenças culturais, sociais e geográficas, bem como os programas de controle existentes. Conclui-se que os programas, de "screening" e de diagnóstico precoce para o câncer de colo uterino tiveram coberturas muito baixas. Considerou-se que o estabelecimento de uma política assistencial e de controle que inclua a prevenção e o diagnóstico precoce para os cânceres de colo uterino e de mama nos programas de assistência à mulher deveria ser uma das prioridades de saúde pública, no Brasil.
\end{abstract}

Descritores: Neoplasias, mortalidade. Saúde da mulher. Fatores de risco.

\section{Introduçăo}

O progressivo aumento das doenças crônicas e degenerativas no Brasil é evidente. As alteraçőes demográficas por que passa a população trarão como consequiência maior quantidade de casos de doenças crônicas dentre as quais se enquadra o câncer. $O$ aumento da vida média, a queda da taxa de fecundidade, as modificações no estilo de vida e a maior exposição a determinados riscos ambientais são fatores que interferem diretamente no aparecimento de um maior número de neoplasias malignas.

Segundo dados do Ministério da Saúde, em 1980 o câncer foi responsável por $10,1 \%$ de todas as mortes registradas no país por causa conhecida 28 . Em 1986, esse percentual foi de 11,2 em ambos os sexos, sendo que no feminino foi de $12.6 \% 29$. Entre as mulheres com mais de 15 anos, $15,6 \%$ dos óbitos tiveram como causa básica neoplasia maligna.

O câncer representou, portanto, a segunda causa de morte no país no sexo feminino, só superado

\footnotetext{
* Serviço de Epidemiologia - Coordenadoria de Programa de Controle de Câncer - Instituto Nacional de Câncer Rio de Janeiro, RJ - Brasil.
}

Separatas/Reprints: G. A. e Silva Mendonça - Praça da Cruz Vermelha, 23 sala 320 - Centro - 20230130 Rio de Janeiro, RJ - Brasil. pelas doenças cardiovasculares. Considerando que o câncer de mama e útero são responsáveis por quase um terço dessas mortes em todo o pais, os programas de prevenção e diagnóstico precoce podem ter grande impacto neste quadro.

A busca de estratégias comuns com aproveitamento dos recursos existentes voltados à assistência integral à saúde da mulher é fundamental dentro de uma política de controle do câncer.

O objetivo do presente trabalho é mostrar, pela análise de dados estatísticos de mortalidade, a importância do câncer como causa de morte e morbidade no país, para a população feminina.

\section{Ocorrência de câncer na população feminina}

A análise dos dados de mortalidade permite o reconhecimento do problema em todo o território nacional, uma vez que o subsistema de mortalidade do Ministério da Saúde apresenta dados que estimam uma cobertura de $75 \% 29$. As Regiőes Norte, Nordeste e Centro-Oeste ainda contam com problemas de cobertura e sub-registro: já as Regiōes Sudeste e Sul apresentam coberturas que se aproximam de $100 \%$. A Tabela 1 mostra a mortalidade proporcional em mulheres segundo principais grupos de causa em todo o país, no ano de 1986. A primeira causa de óbito foi decorrente de doenças do aparelho circulatório com um percen- 
Tabela 1. Número de óbitos e mortalidade proporcional em mulheres segundo principais grupos de causa, Brasil, 1986.

\begin{tabular}{lcc}
\hline Causa (capítulos da CID-9*) & $\begin{array}{c}N^{2} \\
\text { óbitos }\end{array}$ & $\begin{array}{c}\text { Mortalidade } \\
\text { Proporcional }\end{array}$ \\
\hline Doenças aparelho circulatório & 99.649 & $38,1 \%$ \\
Neoplasmas & 33.527 & $12,8 \%$ \\
Doenças aparelho respiratório & 28.338 & $10,8 \%$ \\
Doenças infecciosas e parasitárias & 21.528 & $8,2 \%$ \\
Algumas afecçőes originadas no & & \\
período perinatal & 18.974 & $7,3 \%$ \\
Causas externas & 17.893 & $6,8 \%$ \\
Todas as causas & 33.362 & $100 \%$ \\
\hline
\end{tabular}

- Classificacão internacional de doenças, lesões e causas de óbitos, 9a Revisāo, 1979.

Fonte: Estatisticas de Mortidade - Brasil, 1986²0.
Tabela 2. Mortalidade por câncer em mulheres segundo principais localizaçōes anatómicas, Brasil, 1986.

\begin{tabular}{lcc}
\hline Localização anatómica & (CID-9) $^{*}$ & Freqúéncia relativa \\
\hline mama & 174 & $14,3 \%$ \\
estômago & 151 & $9,2 \%$ \\
colo de útero & 180 & $7,7 \%$ \\
corpo de útero+ & & \\
útero s/ especif. & $179+182$ & $6,6 \%$ \\
colon e reto & $153+154$ & $6,4 \%$ \\
pulmão & 162 & $6,2 \%$ \\
leucemias & 169 & $4,6 \%$ \\
pâncreas & 157 & $3,4 \%$ \\
todas as outras & - & $50,6 \%$
\end{tabular}

- Classificação Internacional de Doenças, Lesões e Causas de Óbitos, $9^{\mathrm{a}}$ Revisão, 1979.

Fonte: Estatísticas de Mortidade - Brasil, 1986².

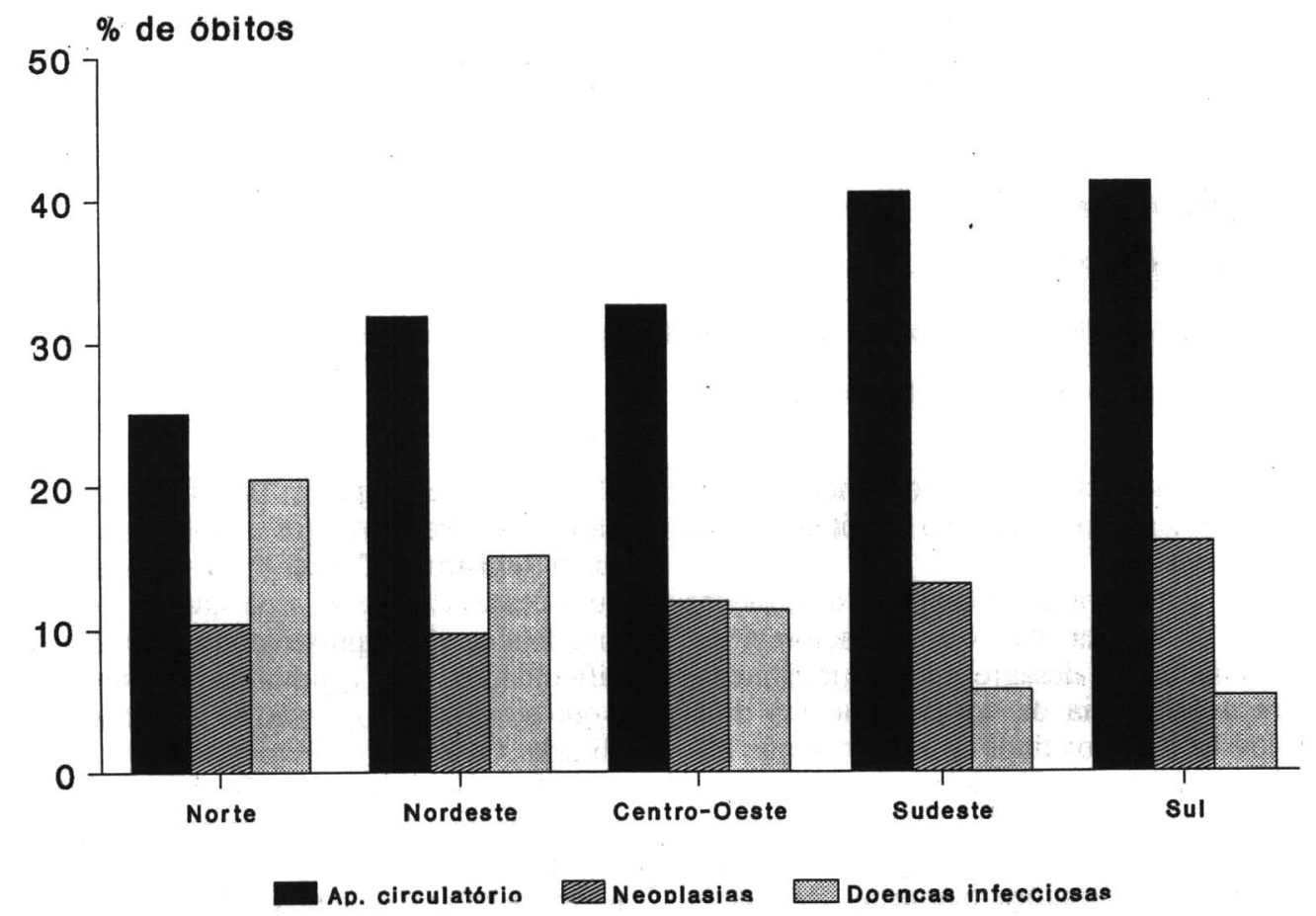

Figura 1. Mortalidade proporcional por principars grupos de causas em mulheres segundo regizes brasileiras, 1986.

Fonte: Estatísitcas de Mortalidade - 198629.

tual de $38,1 \%$. Em segundo lugar encontram-se os neoplasmas $(12,8 \%)$, seguidos das doenças do aparelho respiratório $(10,8 \%)$ e das doenças infecciosas e parasitárias $(8,2 \%)$. As causas externas representam a sexta causa com um percentual de 6,8 .

Ressalva deve ser feita à quantidade ainda importante dos óbitos classificados como causa desconhecida $(21,6 \%$ no sexo feminino em todo o país), o que reflete em última instância a ineficácia da assistência médica e a qualidade dos registros médicos. Na Região Nordeste, os óbitos assim classificados perfizeram $47,6 \%$ do total, o que merece especial atenção.
Analisando-se a mortalidade no sexo feminino, segundo macroregiōes brasileiras, é possível notarse padrões distintos na frequiência relativa dos óbitos segundo esses grupos de causa (Fig. 1). As doenças cardiovasculares apresentam uma frequiência maior nas Regioes Sudeste e Sul, ao passo que as doenças infecciosas são mais freqüentes nas Regiōes Norte e Nordeste, nas quais foram a segunda causa de morte. A Região Sul foi a que apresentou um maior índice de mortes por neoplasias $(15,2 \%)$ e a Região Nordeste o mais baixo $(7,8 \%)$.

Entre as mulheres, o câncer de mama foi o principal tipo de neoplasia malígna que levou ao óbito. 


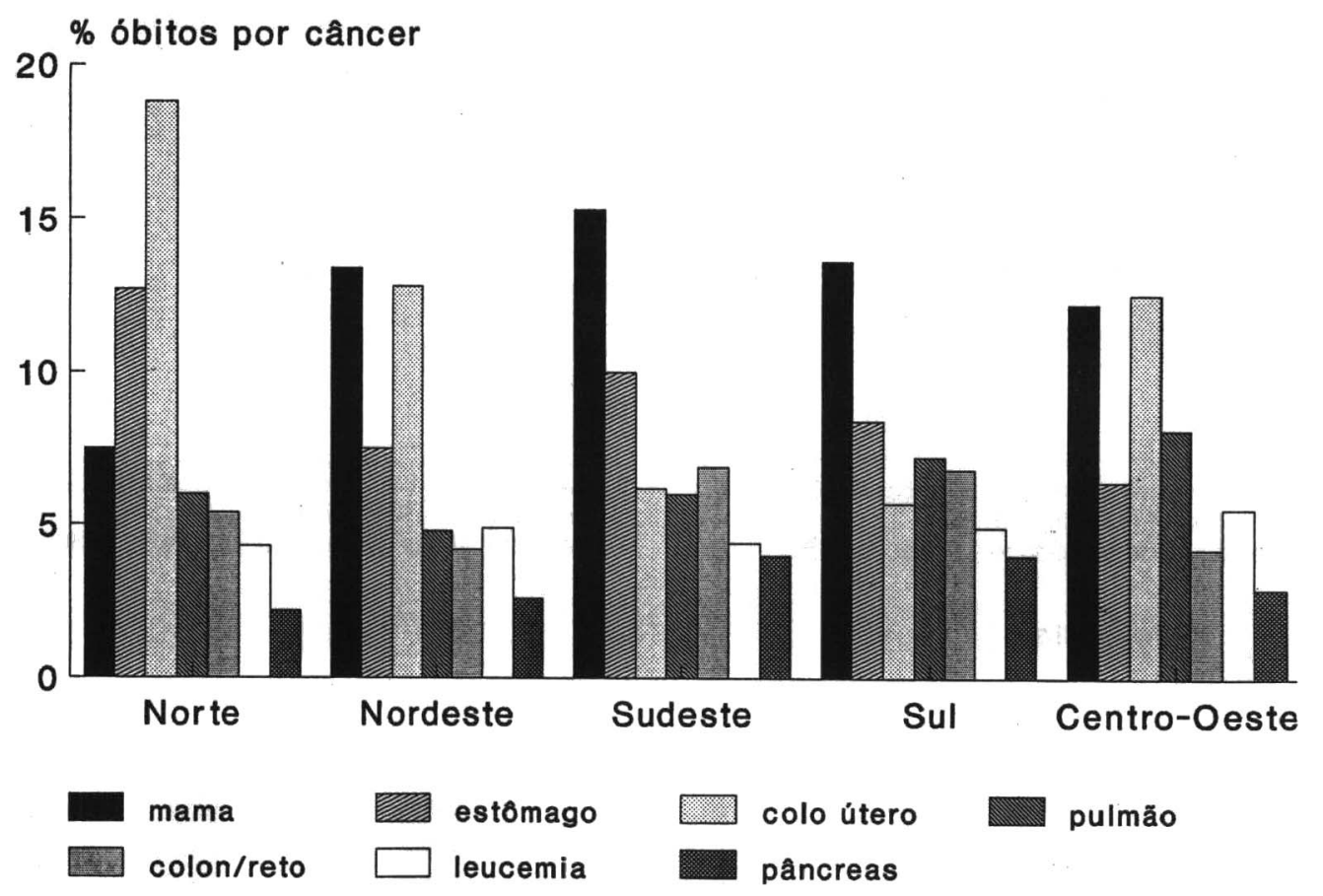

Figura 2. Morialidade proporcional segundo localização anatômica de cânceres mais freqüentes em mulheres, Brasil, 1986.

Fonte: Estatísticas de Mortlidade - Brasil, $1986^{29}$.

Em segundo lugar encontra-se o câncer de estômago e em terceiro o de colo de útero, como mostra a Tabela 2.

A Figura 2 mostra as principais localizaçōes anatômicas de câncer nas cinco macroregiōes. Quando os dados são desagregados regionalmente fica clara a existência de comportamentos diferentes entre os diversos tipos de câncer, o que traduz distintas condiçōes em cada região. Chama a atenção que o câncer de colo de útero foi a primeira causa de óbito por câncer nas regiơs Norte e Centro-Oeste. Nas Regiōes Nordeste, Sudeste e Sul o câncer de mama mostrou-se mais importante. $O$ câncer de estômago foi o segundo mais importante na Região Norte.

Estudos com séries históricas em câncer realizados no Rio de Janeiro, São Paulo e Rio Grande do Sul evidenciaram que dentre os principais tipos de câncer apenas o de estômago, nos dois sexos, mostra tendência a declínio da mortalidade (Mendonça ${ }^{24}$, 1991; Fonseca ${ }^{14}$, 1992; Daudt e col. ${ }^{8}$, 1992). Em São Paulo, entre 1970 e 1989, e no Rio de Janeiro, no periodo de 1979 a 1986 , a mortalidade por câncer de colo uterino e de mama tendeu à estabilidade. No Rio Grande do Sul foi verificado um acréscimo das taxas de mortalidade entre 1977 e 1987.

A incidência de câncer no Brasil pode ser co- nhecida em seis capitais: Belém, Fortaleza, Recife, São Paulo, Porto Alegre e Goiânia, as quais possuem Registro de Câncer de Base Populacional. Alguns desses Registros, especialmente o de Recife e São Paulo, vêm apresentando, nos últimos anos, dificuldades para a continuidade de suas atividades, o que prejudicou a geração de informação mais atualizada. Outros dois Registros mais recentes, em Goiânia e Fortaleza, trabalham dados após 1987 e se acham em fase de aprimoramento do sistema.

Os dados dos quatro Registros brasileiros em torno de 1980 são comparados com as cifras internacionais, na Tabela 3.

Pode ser notado, assim, que os coeficientes gerais de incidência por câncer no sexo feminino em São Paulo e Porto Alegre situam-se em padrões elevados em relação a outras regiơes do Mundo ${ }^{38}$. Os dados de Recife e Fortaleza mostram ser intermediários, porém, superiores aos de outros países da América Latina.

Comparando-se os dados dos registros brasileiros entre si observa-se que eles expressam diferentes comportamentos ${ }^{25}$ que, de certa forma, também foram marcados na análise da mortalidade. A incidência de câncer em São Paulo e Porto Alegre acompanha a tendência internacional, ou seja, com um predomínio do sexo masculino. Já em Recife, Fortaleza, Belém e Goiânia, capitais onde a incidên- 


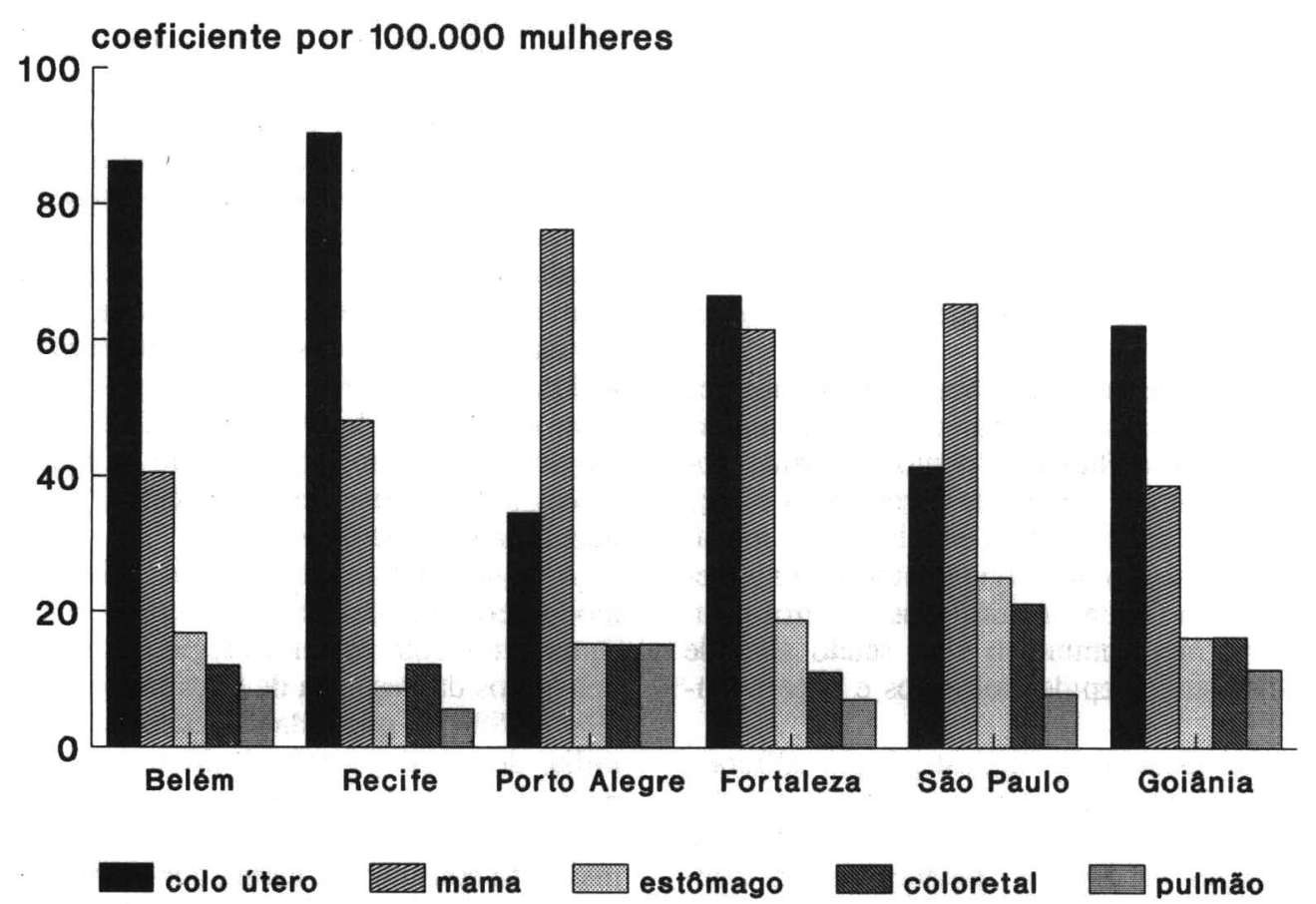

Figura 3 . Incidência* dos cânceres mais freqüentes em mulheres em algumas capitais brasileiras, 1980 - 1988. * Por 100.000 mulheres padronizada pela população mundial. Fonte: Câncer no Brasil: dados dos registros de base populacional?

Tabela 3. Incidência de câncer entre mulheres em quatro capitais brasileiras e de algumas regiōes do mundo, 1970-1980.

\begin{tabular}{lc}
\hline Regiāo* & $\begin{array}{c}\text { Coeficiente de Incidência } \\
\text { por 100.000 mulheres"* }\end{array}$ \\
\hline Havaí (1973-77) & 334,7 \\
Suiça, Genebra (1973-77) & 225,1 \\
Nova Zelândia, Maoris (1972-76) & 302,1 \\
BRASIL, SÃO PAULO (1978) & 277,3 \\
EUA, Connecticut (1973-77) & 257,3 \\
Canadá, Ontário (1973-77) & 233,3 \\
Finlândia (1971-76) & 172,5 \\
China, Shangai (1975) & 158,8 \\
Inglaterra, Oxford (1974-77) & 195,6 \\
BRASIL, P. ALEGRE (1979-80) & 176,9 \\
Israel (1972-76) & 223,0 \\
Japão, Osaka (1973-77) & 137,2 \\
Espanha, Navarra (1973-77) & 145,5 \\
BRASIL, RECIFE (1972-77) & 228,6 \\
BRASIL, FORTALEZA (1978-80) & 223,6 \\
Colômbia, Cali (1972-76) & 212,5 \\
Jamaica, Kingston (1973-77) & 165,5 \\
Cuba (1973-77) & 147,0 \\
India, Poona (1973-77) & 135,6 \\
Senegal, Dacar (1969-74) & 68,0 \\
\hline * Incluídas as regiōes de maiores $\theta$ menores valores re- \\
gistrados.
\end{tabular}

cia de câncer de colo de útero é alta, houve uma inversão deste padrão. A Figura 3 mostra os coeficientes de incidência padronizados pela população mundial para as principais localizaçðes anatômicas nos seis registros de base populacional.

Na presente análise o câncer de pele foi considerado em separado seguindo o modelo convencional de descrição internacional, onde as neoplasias malignas de pele não entram no compto total devido à baixa malignidade da maioria dos casos.

As neoplasias malignas de colo de útero*, mama e estômago foram as três mais importantes. A incidência de câncer de colo de útero em Recife e Belém mostram os mais altos índices mundiais ${ }^{39}$. O câncer de mama em Porto Alegre, capital que apresenta o mais alto coeficiente dentre as outras cinco capitais ( 76,2 por 100.000 mulheres), é tão incidente quanto em algumas cidades americanas. Em São Paulo e Fortaleza a incidência de câncer de mama, também é muito alta se comparada ao quadro mundial.

O câncer de estômago entre mulheres em São

- Considerado aqui os casos de câncer de colo de útero (CID-9 $=180$ ) mais os casos referentes ao útero, porção não especificada, (CID-9 $=179$ ) pois estudos mostraram que em São Paulo $85 \%$ dos óbitos codificados como 179 se referiam, na realidade, a câncer de colo de útero ${ }^{33}$. 
Paulo está entre as quatro regiðes do munido de mais alta incidência (25,1 por 100.000 mulheres), só sendo superada pelos coeficientes do Japão, Cali, Colômbia e Costa Rica. A incidência de câncer de pulmão entre a população feminina de Porto Alegre é comparável a de países desenvolvidos como Canadá e Dinamarca.

\section{Fatores de risco para os cânceres mais freqüentes}

São grandes as interrogações sobre os agentes etiológicos implicados no aparecimento da maioria das neoplasias malignas. É ponto de partida, porém, que a etiologia do câncer é complexa e hoje são conhecidos vários fatores envolvidos no mecanismo da carcinogênese. Alguns fatores de natureza intrínseca já foram identificados e outros relacionados ao meio ambiente vêm sendo alvo de numerosos estudos epidemiológicos e experimentais.

O grande crescimento industrial que sofreram os centros urbanos brasileiros nas últimas décadas traz, como conseqüência direta, maior exposição da população a agentes potencialmente cancerígenos. As taxas de ocorrência de câncer refletem hoje resultados de exposições ocorridas em décadas passadas, momento em que a produção de substâncias cancerigenas era inferior à atual. É esperado, assim, que as neoplasias malignas traduzirão em um futuro próximo todas estas modificações sociais, econômicas e culturais através de suas taxas de incidência.

Para determinados tipos de câncer é possivel identificar fatores que estão associados ao seu aparecimento. Muitos estudos têm sido conduzidos nesse sentido e para algumas neoplasias, em especial, os processos etiológicos já são largamente aceitos. Cabe, portanto, enfatizar os fatores que se relacionam com os principais tipos de câncer e iniciar a discussão de como eles se distribuem e se comportam na população brasileira feminina.

\section{Câncer de mama}

Sabidamente, os principais fatores de risco para o câncer de mama são de natureza biológica e dizem respeito à história reprodutiva e familiar da mulher $^{31}$. A importância, contudo, dos fatores ambientais na etiologia do câncer de mama é clara quando se observa as alteraçōes das taxas em populações migrantes. As taxas de ocorrência entre imigrantes europeus, nos Estados Unidos, aumentaram mais rapidamente do que as de imigrantes japoneses e chineses ${ }^{37}$. A correlação positiva entre nível socioeconômico e a ocorrência de câncer de mama têm sido descrita amplamente. No Brasil, estudo baseado em dados de mortalidade das capi- tais brasileiras comprova esta relaçãos. Outro indicador indireto desta relação pode ser conferido pelas taxas superiores de mortalidade por câncer de mama na Região Metropolitana do Rio de Janeiro, se comparadas às do interior do Estado ${ }^{24}$.

Provavelmente os fatores que mais pesam nessa associação dizem respeito à dieta e a história obstétrica. A associação entre câncer de mama, bem como de colo e reto com a ingestão de proteína animal e consumo de gorduras foi fortalecida após 1970 por Armstrong ${ }^{3}$ e Doll ${ }^{10} \mathrm{com}$ base em dados de 30 países. Estudo epidemiológico, conduzido recentemente na Itália, mostra associação positiva entre ingestão gordurosa e neoplasia maligna de mama ${ }^{9}$.

A dieta da população brasileira é inadequada de modo geral e pode variar de acordo com o poder aquisitivo e com costumes regionais. Segundo dados brutos da Pesquisa de Orçamentos Familiares, $1987 / 1988^{16}$, a quantidade anual de consumo familiar de carne e gorduras difere muito entre cidades das Regióes Nordeste e Norte e Sul. Entre as cidades estudadas, Porto Alegre destaca-se pelo mais alto consumo de carne; coincidentemente é a capital que apresenta os mais altos índices de câncer de mama.

Em que pese os fatores de natureza reprodutiva, é sabido que a primeira gestação em idade mais avançada aumenta o risco de ter câncer de mama ${ }^{21}$. A condição atual da mulher brasileira aproxima-se da encontrada em países desenvolvidos. Dados preliminares do último censo mostram um índice de fecundidade inferior a 3 filhos por mulher em idade produtiva e um crescimento populacional de $1,8 \%$ em $1991^{17}$. Os reflexos dessas alteraçōes demográficas, muito provavelmente, se traduzirão nas taxas de câncer de mama em um futuro próximo.

O fumo vem sendo apontado também como fator que pode estar associado ao risco de ter câncer de mama. Recentemente Palmer e col. ${ }^{32}$ concluíram, através de estudos caso-controle, que esta associação foi mais forte em mulheres que tinham o hábito de fumar na adolescência. Embora pareça ter havido tendência à diminuição do hábito de fumar entre os adolescentes, em algumas capitais brasileiras no final da década de $1980^{7}$, tal hábito entre os jovens da década de 70 era bastante praticado e incentivado pela propaganda de cigarros.

\section{Câncer de colo de uterino}

O câncer de colo uterino está fortemente associado à atividade sexual, mais especificamente, ao número de parceiros e à idade da primeira relação sexual. Existem fortes evidências de que os agentes infecciosos sexualmente transmissíveis 
estão envolvidos na etiologia deste tipo de câncer. $O$ virus do Herpes simples tipo II por mais de vinte anos esteve cotado como o principal responsável dentre os demais. Nos últimos anos, maior atenção vem sendo dada ao papilomavírus humano (HPV) ${ }^{3}$. Embora em nivel experimental o potencial oncogênico do HPV seja evidente, em nível epidemiológico os estudos existentes são altamente sugestivos mas não conclusivos ${ }^{30}$.

Restrepo e col. ${ }^{34}$ relacionam as diferenças de incidência encontradas entre países da América Latina ao grau de desenvolvimento econômico da população e a oferta de serviços de saúde. Em países europeus e da América do Norte os programas de "screening", através da citologia vaginal, têm tido impacto na redução das taxas de mortalidade e incidência ${ }^{11,19,22}$. O desenvolvimento progressivo desta patologia dura em média 10 a 20 anos, o que permite que as açőes de prevenção do carcinoma invasor através da detecção, diagnóstico e tratamento das lesões precursoras tenham êxito de cura de quase $100 \%^{2}$.

A análise de tendência das taxas de mortalidade por essa neoplasia maligna não mostrou variação importante, nesta última década, nos Estados de São Paulo, Rio de Janeiro e Rio Grande do Sul onde se estudou o problema ${ }^{14,24}$ 29. Este fato reforça a idéia de que os programas preventivos levados de país até o momento foram muito pouco efetivos. No Rio de Janeiro, por exemplo, observou-se, inclusive, que as taxas de mortalidade por câncer no colo uterino em mulheres residentes na área metropolitana são similares àquelas de mulheres habitantes no interior do Estado. Tal quadro não seria esperado se o controle tivesse algum nível de eficiência, uma vez que é no Município do Rio de Janeiro e adjacências que se concentra a maior parte de serviços de saúde do Estado.

Outros fatores tais como o uso de contraceptivos orais e fumo também estão implicados no aparecimento do câncer de colo uterino ${ }^{30,40}$. Gram e Stlsberg ${ }^{18}$, a partir de estudo prospectivo entre 1980 e 1989 concluíram que o hábito de fumar está relacionado à neoplasia cervical intraepitelial grau III e ao câncer de colo de útero.

\section{Câncer de estômago}

A mortalidade por câncer de estômago vem sofrendo uma queda em todo o mundo nos últimos cinqüenta anos ${ }^{20}$. Este declínio é decorrente de uma diminuição da incidência, uma vez que a letalidade ainda é alta em função de um tratamento que não evoluiu muito nesses anos. Segundo Doll ${ }^{10}$, esta redução de incidência foi, basicamente, reflexo de alterações de exposição aos dois principais fatores cancerigenos: a preservação inadequada dos alimentos e o uso excessivo de sal.

No Japão, país que apresenta os mais altos índices de câncer de estômago, a partir de 1967 identificou-se diminuição na mortalidade ${ }^{35}$. Tal situação é fruto do resultado dos programas de "screening" em massa que possibilitam o tratamento em estágios iniciais da doença ou em condições pré-malignas. Esses programas são de custo elevadíssimo pois necessitam de procedimentos que envolvem exames radiológicos em larga escala, o que os tornam inviáveis em países subdesenvolvidos.

No sexo feminino, como também no masculino, o câncer de estômago está entre os mais concorrentes tipos enquanto mortalidade e morbidade no Brasil. São Paulo apresentou coeficientes em 1980 que, dentro do painel mundial, se colocavam entre as quatro regiōes de mais alta incidência em ambos os sexos. Contudo, a queda da mortalidade por este tipo de câncer também foi verificada no Brasil, mesmo em regiöes mais carentes como Belém ${ }^{6}$. É esperado, porém, que esta queda não tenha se dado de forma homogênea em todo o país.

No Estado do Rio de Janeiro, por exemplo, o declínio das taxas de mortalidade foram mais elevadas na região metropolitana do que no interior do Estado ${ }^{24}$. A utilização de refrigeradores a partir de 1950 , no Brasil, deve ter tido influência na diminuição do câncer de estômago. A maior dificuldade de acesso a este eletrodoméstico nas zonas rurais pode ser responsável pelas cifras da doença mais altas no interior do país.

\section{Câncer de pulmäo}

Vários estudos têm mostrado que o risco de adoecer por câncer de pulmão é maior em áreas urbanas do que em áreas rurais ${ }^{12,23,36}$. Tal condição é atribuída ao fato de que o hábito de fumar é mais prevalente em áreas urbanas em função de um maior grau de poluição atmosférica.

No Brasil, em 1989, existiam 30,2 milhões de fumantes, entre os habitantes maiores de 15 anos o que corresponde a $32,6 \%$ da população segundo dados do Instituto Brasileiro de Geografia e Estatística (IBGE) ${ }^{15}$. As mulheres mostraram uma proporção maior do que os homens até os 50 anos, o que sugere que elas devem estar incorporando o hábito de fumar mais precocemente ${ }^{27}$. Entre $30 \mathrm{e}$ 49 anos, faixa etária da população que mais fuma, $42,4 \%$ dos fumantes eram do sexo masculino e $43,1 \%$ do sexo feminino. Não é possível se fazer uma correlação direta, mas chama a atenção que o aumento do coeficiente de mortalidade específico por câncer de pulmão em todo o país sofreu, justamente, aumento mais marcado no sexo feminino 
entre os anos de 1979 e 1986, passando de 4,1 para 4,4 por 100.000 mulheres $^{27}$.

A prevalência do tabagismo no final da década de 1980 sofreu uma inversão em relação a zona rural e urbana do Brasil. Segundo dados da Pesquisa Nacional sobre Saúde e Nutrição ${ }^{15}$, a prevalência do hábito de fumar na zona rural foi de $26,4 \%$ enquanto na urbana foi de $23,2 \%$. Isto pode explicar, talvez a tendência ascendente da mortalidade por câncer de pulmão verificada no interior do Estado do Rio de Janeiro, tanto no sexo masculino quanto feminino, entre 1979-1986, o que não aconteceu na Região metropolitana do mesmo Estado ${ }^{24}$.

\section{Comentários Finais}

Diante do exposto, pode-se concluir que as neoplasias malignas, em especial os tumores de mama e colo uterino, exercem um peso importante na morbidade e mortalidade das mulheres brasileiras.

A experiência internacional tem mostrado que atividades de controle, conduzidas de forma adequada, seriam capazes de reduzir a ocorrência de câncer de colo uterino em mais de $60 \%$ e a mortalidade por câncer de mama entre mulheres de $50 \mathrm{a}$ 69 anos, em $40 \% 41$.

A American Cancer Societyl é enfática em afirmar que nos Estados Unidos, em 1992, 100.000 pacientes a mais com câncer sobreviveriam se a doença tivesse sido diagnosticada precocemente. Entre estes estariam os casos de câncer de mama, língua, boca, cólon, reto, colo de útero, próstata, testículo e melanoma. A sobrevida, que para estes casos foi de $33 \%$ em cinco anos, chegaria a $89 \%$. O câncer de colo uterino exige, para sua prevenção e diagnóstico precoce, procedimentos simples e de baixo custo que poderiam ser realizados em toda a rede pública nacional. Até o momento, os levantamentos existentes sobre os exames citologicos vaginais mostram coberturas baixíssimas ${ }^{26}$. Em 1987, esta cobertura no Brasil foi de $8 \%$ das mulheres acima de 20 anos, enquanto que a Organização Mundial de Saúde estabelece uma cobertura mínima de $85 \%$.

Avaliação feita do programa de controle do câncer de colo de útero, realizada em 1984 dentro da rede pública, evidenciou que as Secretarias de Saúde realizaram o exame preventivo em apenas $7 \%$ de suas unidades básicas e o INAMPS não tinha esta informação registrada ${ }^{2}$. Ainda nesta avaliação foi possível perceber a grande predominância de diagnósticos de carcinoma invasivo sobre o estágio in situ, o que não se esperaria em programas de detecção precoce. Em última análise, este padrão indica que o atendimento é voltado para uma clientela que procura os serviços de saúde já em fase sintomática e ocorre de forma mais acentuada em áreas de maior incidência da doença ${ }^{13}$.

Concomitante aos programas de "screening" para o câncer de colo uterino, atenção deve ser dada aos voltados para o câncer de mama. Ilustra bem o quadro brasileiro, a situação de Fortaleza, em que a ocorrência destes dois tipos de câncer apresenta taxas quase que similares mesmo em se tratando de uma capital em região caracteristicamente carente.

Esforços no sentido de se integrar ações voltadas à saúde da mulher, que encarem conjuntamente a questão do câncer ginecológico, devem tornar-se rotina da prática diária dos serviços de saúde. Tais açð̄es, incorporando o diagnóstico precoce do câncer de mama, dependeriam muito mais de um treinamento de pessoal qualificado para a detecção a partir do exame físico do que de tecnologia de alto custo. Estudos realizados no Canadá mostram que a sensibilidade do exame físico feito por profissional treinado para rastreamento das neoplasias mamárias em programas ampliados é superior à da mamografia 4 .

Situaçōes tais como a desarticulação entre os diversos níveis de assistência à saúde, ociosidade da rede pública, falta de programação e avaliação das ações impedem que qualquer programa assistencial ou preventivo possa ter melhores resultados.

Faz-se, portanto, premente o estabelecimento de uma política assistencial e preventiva que inclua a questão do câncer ginecológico ao programa de assistência integral à mulher.

MENDONÇA, G. A. e S. [Cancer among women in Brazil]. Rev. Saúde Pública, 27: 68-75, 1993. This study seeks to show the importance of cancer as a cause of morbidity and mortality among Brazilian women. Mortality data from the Health Ministry and incidence data from the six Brazilian Population Based Cancer Registries (Belém, Fortaleza, Recife, S. Paulo, Porto Alegre e Goiânia) were used. Cancer accounted for $15.5 \%$ of all deaths in women above fifteen years of age, in 1986. Breast and uterine cancer were responsible for some $30 \%$ of these deaths. International comparisons show that Recife and Belém had the highest incidence rates from cervical cancer in the world. The incidence of breast cancer in Porto Alegre, S. Paulo, Fortaleza and Goiânia approximated to the figures for regions of high incidence such as the United States and some European countries. Risk factors in leading female cancers are discussed in the light of cultural, social and geographical differences, as well as the development of screening programs. It is concluded that screening and earlydetection programs for cervical and breast cancer had very low coverage in the country. The insertion of actions for the prevention and early diagnosis of cervical 
and breast cancers in women's health care programs should be considered one of the priorities for public health in Brazil.

Keywords: Neoplasms, mortality. Women's health. Risk factors.

\section{Referências Bibliográficas}

\section{AMERICAN CANCER SOCIETY. Cancer facts and figures} - 1992. Atlanta, 1992.

2. AQUTNO, E. M. L. et al. Situação atual da deteçãa precoce do câncer cérvico-uterino no Brasil. Cad. Saúde Pública, 2: 53-65, 1986.

3. ARMSTRONG, B. et al. Human papillomavirus and cervical cancer. Lancet, 2: 756-7, 1988.

4. BAINES, C. J. et al. Physical examination: its role as a single screening modality in the Canadian National Breast Screening Study. Cancer, 63: 1816-22, 1989.

5. BUSTAMANTE, M. T. Mortalidade por câncer no Estado do Rio de Janeiro, 1979/81: um estudo da distribuição geográfica. Rio de Janeiro, 1992. [Dissertação de Mestrado. - Universidade Estadual do Rio de Janeiro].

6. CÂNCER: mortalidade no Brasil. RADIS/Dados FIOCRUZ, Rio de Janeiro (15): 1991.

7. CARLINI, E. A. et al. II Levantamento nacional sobre uso de psicotrópicos em estudantes de $1^{9}$ e $2^{q}$ graus. CEBRID/Escola Paulista de Medicina, 1989.

8. DAUT, A. W. et al. Estudo da mortalidade por doenças tabaco-dependente. [Apresentado Conferência Mundial sobre Tabaco ou Saúde, à 13² Buenos Aires, 1992]

9. DÁVANZO, B. et al. Fats in seasoning and breas cancer risk: an Italian case-control study. Eur. J. Cancer, 27: 420-3, 1991.

10. DOLL, R. Are we winning the figth against cancer? An epidemiological assessment. Eur. J. Cancer, 26: 611-21, 1990.

11. DRAPER, G. J. \& COOK, G. A. Changing patterns of cervical cancer rates. Br. Med. J., 287: 510-2, 1983.

12. FACCHINI, U. et al. Geographical variation of cancer mortality in Italy. Int. J. Epidemiol., 13: 53-4, 1985.

13. FAERSTEN, E. Câncer cérvico-uterino e lesões precursoras: aspectos da ocorrência, controle e vigilância epidemiológica no Brasil. Rio de Janeiro, 1987. [Dissertação de Mestrado - Universidade Estadual do Rio de Janeiro].

14. FONSECA, L. A. M. Mortalidade por câncer no Estado de São Paulo. Oncol. Atual, 2: 6-14, 1992.

15. FUNDAÇÃO IBGE. Pesquisa nacional sobre nutrição; dados preliminares. Brasília, 1991.

16. FUNDAÇÃO IBGE. Pesquisa de orçamentos familiares 1987/88: consumo alimentar domiciliar "per capita". Rio de Janeiro, 1990

17. FUNDAÇÃO IBGE. Resultados parciais do censo - 1991; Tabulação especial. Rio de Janeiro, 1992.*

18. GRAM, I. T. \& STLSBERG, H. Cigarette smoking and the incidence of cervical intraepithelial neoplasia, grade III and cancer of the cervix uteri. Am. J. Epidemiol., 135: $341-6,1992$.
19. GUZICK, D. S. Efficacy of screening for cervical cancer, a review. Am. J. Public Health, 68: 125-34, 1978.

20. HOWSON, C. P. et al. The decline in gastric cancer: epidemiology of an implaned triumph. Epidemiol. Res., 8: $1-27,1986$

21. JACOBSEN, B. K. \& LUND, E. Level of education, use of oral contraceptives and reproductive factors: the TROMSO study. Int. J. Epidemiol., 19: 967-70, 1990.

22. LYNGE, E. Regional trends in incidence of cervical cancer in Denmark in relation to local smear taking activity. Int. J. Epidemiol., 12: 405-13, 1983.

23. MATOS, E. L. et al. Patterns of cancer mortality in Argentina. Int. J. Epidemiol., 19: 860-70, 1990.

24. MENDONÇA, G. A. S. Mortalidade por câncer no estado do Rio de Janeiro, 1979/86. Rio de Janeiro. 1991. [Dissertação de Mestrado - Universidade Estadual do Rio de Janeiro].

25. MINISTÉRIO DA SAÚDE. Câncer no Brasil: dados dos registros de base populacional. Rio de Janeiro, Instituto Nacional de Câncer, 1991.

26. MINISTÉRIO DA SAUDE. Controle do câncer cérvicouterino e da mama. Brasilia, 1989.

27. MINISTÉRIO DA SAÚDE. Controle do tabagismo: um desafio. Rio de Janeiro, Instituto Nacional de Câncer, 1992.

28. MINISTÉRIO DA SAUDE. Estatísticas de mortalidade • Brasil: 1980. Brasília, 1983.

29. MINISTÉRIO DA SAULDE. Estatísticas de mortalidade • Brasil: 1986. Brasília, 1991.

30. MUNÕZ, N. et al. Does human papillomavirus reduce cervical cancer? The state of epidemiological evidence. $\mathrm{Br}$. J. Cancer, 57: 1-5, 1988.

31. PAGE, H. S. \& ASIRE, A. J. Cancer rates and risks. National Institute of Health. Washington, DC, Publication, $\mathrm{n}^{2} 85,1985$.

32. PALMER, R. J. et al. Breast cancer and cigarette smoking: a hypothesis. Amer. J. Epidemiol., 134: 1-13, 1991.

33. PUFFER, R. R. \& GRIFFITH, G. W. Caracteristicas de la mortalidad urbana. Washington. Organización Panamericana de la Salud, 1968. (Publ. cient. 151).

34. RESTREPO, H. E. et al. Epidemiologia y control del cancer del cuello uterino en America Latina y el Caribe. Bol. Of. Sanit. Panam., 102: 578-92, 1987.

35. SEGI, M. \& KURIHARA, M. Cancer mortality for selected sites in 24 countries, (1966-1967). Jpn. Cancer Soc., 6: $98,1972$.

36. SIERRA, R.; PARKIN, D. M.; LEIVA, G. M. Cancer in Costa Rica. Cancer Res., 49: 717-24, 1989.

37. TOMATIS, L. et al. Cancer: causes, occurrence and control. Lyon, 1990. (IARC Scient. Publ. 100).

38. WATERHOUSE, J. et al. Cancer incidence in five continents. $\dot{V}$. Lyon, 1987. (IARC Scient. Publ. 88).

39. WHELAN, S. L. et al. Patterns of cancer in five continents. $V$. Lyon, 1990. (IARC Scient. Publ. 12).

40. WINKELSTEIN JR., W. Smoking and cervical cancer current status: a review. Am. J. Epidemiol., 131: 945-75. 1990.

41. WORLD HEALTH ORGANIZATION. Cancer control programme. Geneva, 1992

Recebido para publicaçâo em 16.7.1992 Reapresentado em 16.12.1992 Aprovado para publicação em 21.12.1992

- Dados Inéditos. 